Ann. Génét. Sél. anim., I972, 4 (4), 593-598.

\title{
OBSERVATIONS SUR LES CARACTERES DE REPRODUCTION DES PRODUITS MALES ET FEMELLES ISSUS D'UN BOUC PORTEUR D'UNE « FUSION CENTRIQUE »
}

\author{
G. RICORDEAU \\ avec la collaboration technique de R. Mollaret et P. Guillimin, \\ Laboratoive de Génétique des Petits Ruminants, \\ Centre de Recherches de Toulouse, I. N.R. A., \\ BP. 12, 31320 Castanet Tolosan
}

\section{RÉSUMÉ}

Dans cette étude sont comparés les caractères de reproduction des produits issus d'un bouc porteur d'une fusion centrique.

Sur r 8 mâles examinés : 4 étaient normaux et fertiles; 14 étaient porteurs, dont I I fertiles, (produisant en moyenne I 7 p. 100 de spermatozoïdes en moins que les 4 normaux), 2 totalement stériles et I partiellement stérile présentant une simple épididymite. Parmi les 3 cas de stérilité, l'un est probablement homozygote sans cornes, alors que les 2 autres sont hétérozygotes, aussi la stérilité peut résulter aussi bien d'une action du gène $P$ que de la fusion centrique, bien que cela soit statistiquement peu vraisemblable pour les 2 hétérozygotes.

Quant aux I 7 chevrettes examinées, 8 étaient normales et ont donné en moyenne $\mathbf{1}, 75$ produit ; sur les 9 porteuses de la fusion une est restée vide, les 8 fécondées n'ayant donné en moyenne que I,37 produit. Le nombre de saillies nécessaires pour obtenir une fécondation a été de 1,25 pour les deux catégories. Ces résultats rendent valables pour les caprins I'hypothèse de GusTAvsson (1969) selon laquelle la mortalité embryonnaire serait plus importante chez les femelles porteuses d'une fusion centrique que chez les femelles normales.

En 1969 et 1970, au Domaine Expérimental de Galle (1 8 - Avord), nous avons utilisé un bouc de race Alpine Saanen, originaire de Suisse et porteur d'une "fusion centrique " à 59 chromosomes déjà signalée par Hulot (I969) et analogue à celle décrite par PADEH et al. (I966) et SoLLER et al., (197 I).

Le déterminisme héréditaire de cette anomalie a été étudié par Popescu (1972) sur les données du troupeau de Galle. Nous nous limiterons donc ici aux résultats concernant les caractères de reproduction des produits porteurs de la fusion et des produits normaux. 


\section{RÉSULTATS}

En 1970, sur les 50 produits obtenus de 23 chèvres, 6 sont morts à la naissance et 3 ont été réformés au cours des 3 premiers mois. En I97I, on a obtenu 8 produits de 5 chevrettes.

Les caryotypes ont été effectués sur 29 produits nés en 1970 (I 8 mâles et II femelles) et sur 6 femelles nées en 1971 .

\section{I. - Observations sur les boucs}

Les I 8 mâles nés du 2-I2-1969 au 30-2-1970 ont été soumis à des contrôles de semence du I9 au 24-IO-I970, pendant 6 jours consécutifs, après une période d'entrainement de ro jours, durant laquelle les boucs devaient effectuer $\mathrm{I}$ saut tous les 2 jours. Le critère retenu pour juger la qualité de la semence fraîche est le nombre total de spermatozoïdes, calculé d'après le volume de l'éjaculat et la concentration.

Les I 8 mâles se classent ainsi :

-4 sont de caryotype normal et fertiles ;

- I4 sont porteurs de l'anomalie soit :

- 2 stériles,

- I 2 fertiles dont $\mathrm{r}$ avec une épididymite droite.

Sur les 2 mâles stériles, l'un est probablement homozygote sans cornes PP (70039), l'autre est hétérozygote (70159) car il est né de mère cornue et porte des cornillons. Ces 2 boucs ont été abattus en février 1972, c'est-à-dire en période de repos sexuel (tabl. I). Du point de vue spermatogenèse, ils présentaient un aspect hétérogène du parenchyme testiculaire avec des tubes d'apparence normale voisinant avec des tubes à peu près vides de cellules germinales, alors que les testicules de boucs fertiles examinés à la même époque ne contenaient que des tubes "actifs"; ils rappellent en cela certains animaux stériles, à caryotype normal, examinés antérieurement (CoRTeel et al., r969). L'activité endocrine des testicules a été estimée par dosage de la testostérone circulante dans le sang jugulaire -3 jours de suite - et de la concentration du tissu testiculaire en testostérone lors de l'abattage. Compte tenu de la saison, cette activité semble à un niveau assez voisin de celui d'animaux normaux contrôlés à la même époque : elle est cependant plus faible pour le 70I59. Si la stérilité du bouc 70039 est vraisemblablement due à son génotype

\section{TABLEAU I}

Observations sur les deux boucs stériles

\begin{tabular}{|c|c|c|}
\hline & Bouc no 70039 & Bouc n 70159 \\
\hline Poids vif $(\mathrm{kg})$ en avril $1971 \ldots \ldots \ldots$ & 55,0 & 43,0 \\
\hline $\begin{array}{l}\text { Poids des testicules à l'abattage, en } \\
\quad \text { février } 1972(\mathrm{~g}) \ldots \ldots \ldots \ldots \ldots \ldots \ldots\end{array}$ & 142 & 125 \\
\hline Diamètre des tubes $\int$ actifs $\ldots . . .$. & 224 & 227 \\
\hline séminifères $(\mu) \quad\{$ vides $\ldots \ldots \ldots$ & 178 & 185 \\
\hline $\begin{array}{c}\text { Testostérone plasmatique }(\mathrm{ng} / \mathrm{ml} \text { de } \\
\text { plasma) } \ldots \ldots \ldots \ldots \ldots \ldots \ldots \ldots \ldots\end{array}$ & $10,4-2,6-1,1$ & $11,7-4,6-1,9$ \\
\hline $\begin{array}{l}\text { Testostérone testiculaire }(\mathrm{ng} / \mathrm{mg} \text { tissus } \\
\text { frais) } \ldots \ldots \ldots \ldots \ldots \ldots \ldots \ldots \ldots\end{array}$ & 65,6 & 38,0 \\
\hline
\end{tabular}


cornage, il est bien difficile d'expliquer celle du 70159: puisque les boucs hétérozygotes sans cornes sont pratiquement tous fertiles (RICORDEAU et al., I972) on peut penser que cette stérilité résulte de l'anomalie caryotypique mais, du fait que les examens histologiques sont comparables à ceux effectués sur les boucs stériles examinés antérieurement, il n'est pas exclu que le bouc 70I 59 - comme le bouc 70152 dont il est question par la suite-soit stérile par suite d'un blocage de l'épididyme consécutif à l'action du gène $\mathrm{P}$ (Lauvergne, I969).

Les $\mathrm{I} 6$ mâles fertiles ont été abattus le 6-4-197x et les testicules disséqués afin de mesurer leur poids et celui des 3 régions de l'épididyme. Le tableau 2 résume les résultats obtenus sur les animaux porteurs et normaux; nous avons mis à part le bouc 7 or 52, également hétérozygote sans cornes, qui présente une simple épididymite et apparaît de ce fait difficilement comparable aux autres. A ro mois, les boucs porteurs de la fusion ont une production de spermatozoïdes inférieure - mais non significativement différente - à celle des boucs normaux : r,882 milliards de spermatozoïdes pour les I 2 mâles porteurs contre 2,267 pour les 4 normaux, soit une différence de $\mathrm{r} 7 \mathrm{p}$. Ioo. A l'abattage - I 4 mois - les poids des testicules et des 3 régions de l'épididyme sont peu différents.

\section{TABLEAU 2}

Comparaison des boucs normaux et porteurs de la fusion

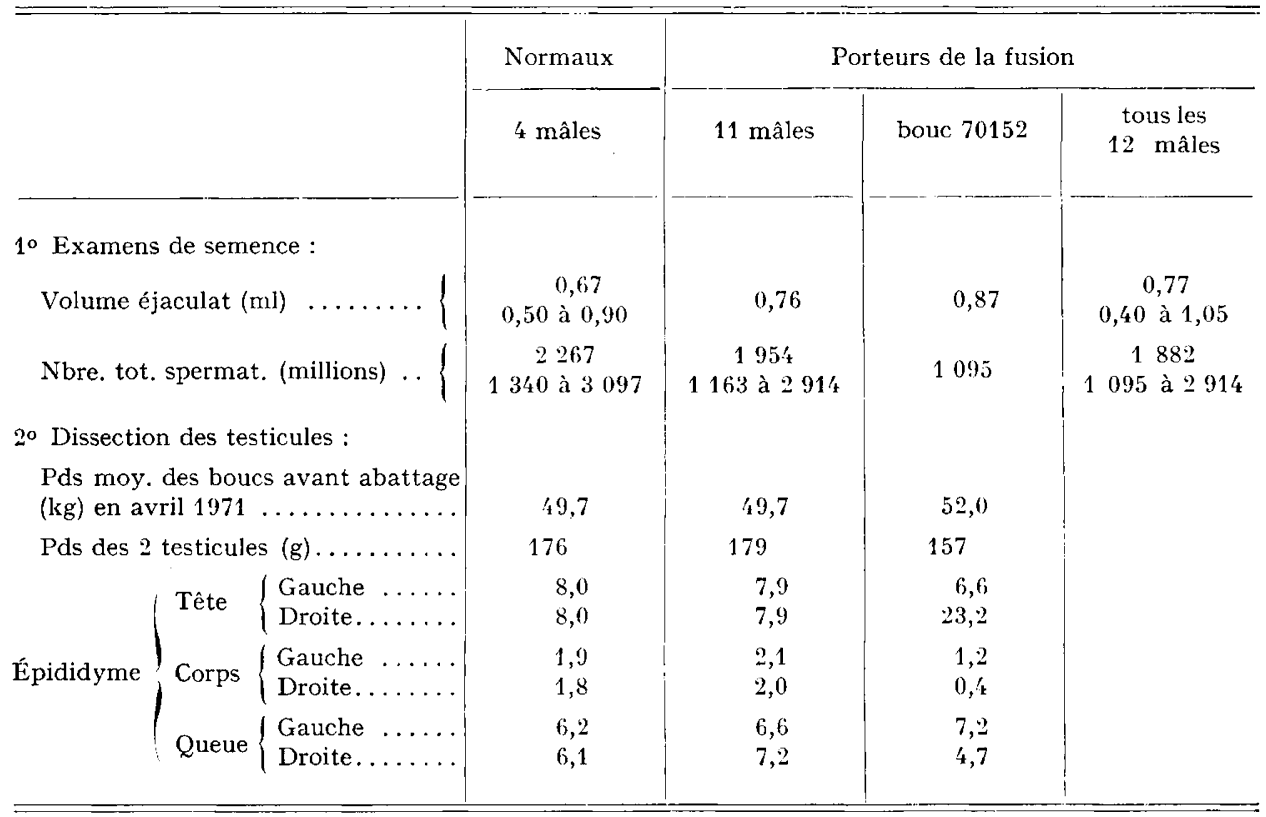

II. - Observations sur les femelles

Sur les I 7 filles conservées pour la reproduction, 9 sont porteuses de la fusion et 8 sont normales.

Ces filles ont été soumises à la saillie pour la I $^{\text {re }}$ fois, à partir de 7 mois, en 1970 et 197 I. Le tableau 3 indique le nombre de saillies réalisées et le nombre moyen de produits par mise bas.

Les 8 chevrettes normales ont toutes été fécondées et ont donné en moyenne 1,75 produit; 
en revanche, sur les 9 chevrettes "porteuses ", I est restée vide et les 8 fécondées n'ont donné que I,37 produit, prolificité significativement inférieure à celle des normales. Le nombre de saillies nécessaires pour obtenir une fécondation a été de 1,25 pour les 2 catégories.

\section{TABLEAU 3}

Fertilité et prolificité des cheurettes issues du même père et soumises à la saillie à 7 mois

\begin{tabular}{|c|c|c|c|c|}
\hline \multirow{2}{*}{ Catégorie des animaux } & \multicolumn{2}{|c|}{8 femelles normales } & \multicolumn{2}{|c|}{9 femelles porteuses de la fusion } \\
\hline & $\begin{array}{l}\text { Nombre de } \\
\text { saillies }\end{array}$ & $\begin{array}{l}\text { Nombre de } \\
\text { produits }\end{array}$ & $\begin{array}{l}\text { Nombre de } \\
\text { saillies }\end{array}$ & $\begin{array}{l}\text { Nombre de } \\
\text { produits }\end{array}$ \\
\hline \multirow{6}{*}{ Nées fin 1969 , début 70} & 1 & 2 & 1 & 2 \\
\hline & 1 & 2 & 3 & 2 \\
\hline & 1 & 2 & 1 & 1 \\
\hline & 1 & 2 & 1 & 1 \\
\hline & 2 & 1 & 1 & \\
\hline & & & 4 & 0 (vide) \\
\hline \multirow{3}{*}{ Nées fin 1970 , début 71\{} & 1 & 2 & 1 & 2 \\
\hline & 2 & 2 & 1 & 1 \\
\hline & 1 & 1 & 1 & 1 \\
\hline $\begin{array}{l}\text { Moy. calculée sur les chevrettes fé- } \\
\text { condées } \ldots \ldots \ldots \ldots \ldots \ldots \ldots \ldots\end{array}$ & 1,25 & 1,75 & 1,25 & 1,37 \\
\hline
\end{tabular}

\section{DISCUSSION}

Chez les ovins, l'anomalie "fusion centrique " affecte la fertilité des mâles et entraîne des cas de stérilité dont on ignore l'importance exacte dans la mesure où de nombreux jeunes mâles "porteurs " sont éliminés avant la période de reproduction (cf. revue bibliographique de BRUÈrE, I969; Bruère et Mills, I97I). Chez les bovins de la race Pie-Rouge Suédoise (S. R. B.), GusTAVSSON (r969) signale que le taux de non-retour à 56 jours des filles issues de taureaux "porteurs " n'est pas significativement différent de celui des filles issues de taureaux "normaux ", alors que le taux de non-retour à 273 jours est significativement plus faible pour les premières.

Notre échantillon de mâles et de femelles est restreint, mais les animaux mis en comparaison sont issus du même père, ont en moyenne le même âge et ont été contrôlés dans des conditions identiques. Chez les mâles, il est difficile de tirer des conclusions précises. En revanche, les résultats obtenus montrent que l'anomalie "fusion centrique " entraîne une baisse significative de la fertilité chez les femelles, ce qui laisse à penser que l'hypothèse de Gustavsson (1969) suivant laquelle la baisse de fertilité des filles issues de taureaux porteurs résulterait d'une augmentation de la mortalité " embryonnaire " s'appliquerait également aux caprins.

Il importe maintenant de vérifier ces observations sur un plus grand nombre d'animaux afin de mesurer de façon plus précise l'importance des pertes dues à cette anomalie et entreprendre, si possible, un dépistage systématique des boucs utilisés pour la reproduction et notamment les boucs à soumettre au testage. Cette anomalie est certainement fréquente chez les caprins : elle a été signalée en Israël et nous l'avons observée en France dans 2 troupeaux totalement indépendants en ce qui concerne l'origine des animaux : à Jouy, I bouc anormal sur 3 examinés HuLor, 1953) ; à Galle, I bouc anormal sur 7 examinés. Il est vraisemblable que cette anomalie 
existe avec la même fréquence dans les troupeaux de ferme, puisque la sélection de l'éleveur ne joue pas dans ce cas. En Suède, par exemple, Gustavsson (I969) a montré que sur I I73 vaches Pie-Rouge, il y avait I 4 p. Ioo d'hétérozygotes et o,34 p. Ioo d'homozygotes. Depuis, les examens réalisés en France ont montré que cette anomalie existait également dans nos populations bovines (Popescu, I97I).

Reçu pour publication en septembre 1972.

\title{
REMERCIEMENTS
}

Cette étude a été réalisée grâce à l'aide de C. P. Popescu pour les examens caryotypiques, de Mme Lise Martinet et de MM. Courot, Corteel et Saumande pour les observations sur les tractus mâles et les dosages de testostérone : nous les remercions sincèrement de leur collaboration.

\section{SUMMARY}

\section{OBSERVATIONS ON THE REPRODUCTION TRAITS}

\author{
OF MALE AND FEMALE PROGENY FROM A HE-GOAT CARRYING CENTRIC FUSION
}

The reproduction traits of progeny from a he-goat carrying centric fusion are compared.

Of 18 males examined, 4 were normal and fertile, I 4 were carriers, I I of which were fertile but producing an average of less (I 4 p. IOo) spermatozoa than the 4 normal and 2 sterile, one of which had a simple epididymite. Sterility probably resulted from action of the $\mathrm{P}$ gene, independent of centric fusion.

As to the $x 7$ young she-goats examined, 8 were normal and gave an average of $\mathrm{I} .75$ products. Of the 9 carriers of centric fusion, one remained sterile, the 8 fertilized giving only an average of I.37 products. The number of matings necessary to obtain a fertilization were 1.25 for both categories. These results indicate that Gustavsson's hypothesis (1969) concerning cows is also valid for goats, namely that embryonic mortality is higher in females carrying centric fusion than in normal females.

\section{RÉFÉRENCES BIBLIOGRAPHIQUES}

BRUÈre A. N., 1969. Male sterility and an autosomal translocation in Romney sheep. Cytogenetics, 8, 209-218.

Bruère A. N., Mills R. A., r971. Observations on the incidence of Robertsonian translocations and associated testicular changes in a flock of New Zealand Romney sheep. Cytogenetics, 10, 260-272.

Corteel J. M., Hulot F., Courot M., Attal J., Philippon A., ig69. Examens morphologiques, caryologiques, physiologiques et pathologiques de boucs stériles sans cornes. Ann. Génét. Sél. anim., $1,34 \mathrm{r}-348$.

Gustavsson I., r959. Cytogenetics, distribution and phenotypic effects of translocation in Swedish cattle. Hereditas, 63, 68-169.

Hulor F., I969. Nouveau cas de fusion centrique chez la chèvre domestique (Capra hircus L.). Ann. Génét. Sélect. anim., 1, I75-176.

Lauvergne J.-J., I969. Progrès des connaissances génétiques sur l'intersexualité associée à l'absence de cornes chez la chèvre d'origine alpine. Ann. Génét. Sél. anim., 1, 403-412.

PAdeh B., Wysoki M., Soller M., I97r. Further studies on a Robertsonian translocation in the Saanen dairy goat. Cytogenetics, 10, 6r-69.

Popescu C. P., r97x. Deux cas nouveaux de fusion centrique chez les bovins. Ann. Génét. Sél. anim. 3, 52I-524. 
Popescu C. P., 1972. Le mode de transmission d'une fusion centrique dans la descendance d'un bouc (Capra hircus L) hétérozygotde. Ann. Génét. Sél. anim. 4, 355-36r.

Ricordeau G., Bouillon J., Hulot F., I972. Pénétrance de l'effet de stérilité totale lié au gène sans cornes $\mathbf{P}$, chez les boucs. Ann. Génét. Sél. anim., 3.

Soller M., Wysoki M., PADeH B., r966. A chromosomal abnormality in phenotypically normal Saanen goats. Cytogenetics, 5, 88-93. 